\title{
LA REGIÓN DE LOS RÍOS COMO PROYECTO POLÍTICO SUBNACIONAL: UNA MIRADA AL PROCESO DE UNA REGIÓN NUEVA
}

\section{THE REGION OF THE RIVERS AND SUBNATIONAL POLITICAL PROJECT: A LOOK AT THE PROCESS OF A NEW REGION}

\author{
José Escaida Navarro ${ }^{1}$ \\ Juan Carlos Miranda ${ }^{2}$ \\ Francisco Mauricio Vega-Duarte ${ }^{3}$
}

\section{Resumen}

La creación de la Región de Los Ríos, viene a ser un caso de proyecto político subnacional, que en contextos de hiperpresidencialismo y centralismo chileno, responde de manera consistente en la generación de instancias regionales de administración de políticas públicas, gobernanza y participación, pero que requiere mayores niveles de profundización de decisiones con autonomía regional, en un contexto de Estado multinivel.

El presente artículo además de hacer una revisión de los antecedentes que dieron origen a la creación de la región, se adentra a describir el rol jugado por los actores políticos, gremiales, sociales, académicos y de la participación ciudadana, así como exploraciones y prospecciones entorno a la consolidación del desarrollo regional sobre la base de dinámicas de descentralización.

Palabras claves: Descentralización, Gobernanza, Participación, Región de Los Ríos, Político subnacional.

\begin{abstract}
The proposed creation of the Región de Los Ríos, it becomes a case of subnational political project, which in the context of hyper and Chilean centralism comes to respond consistently in generating regional bodies of public policy management, governance and participation, but that requires higher levels of deepening regional autonomy decisions in a context of multilevel State.

This article in addition to a review of the background that led to the creation of the region, enters to describe the role played by politics, trade, social, academic and citizen participation actors and explorations and surveys around the consolidation of regional development based on decentralization dynamics.
\end{abstract}

Keywords: Descentralization, Gobernance, Participation, Región de Los Ríos, Subnational political.

\footnotetext{
${ }^{1}$ Sociólogo. Doctor en Sociología. E-mail: joseescaida@uach.cl

2 Postgrado (magíster) en población y Desarrollo. Dr.(c) Economía Aplicada y Análisis Regional. E-mail: jmiranda@uach.cl

${ }^{3}$ Académico. Departamento de Sociología y Ciencia Política UC Temuco. Facultad de Ciencias Sociales. E-mail: covegaduarte@gmail.com
} 


\section{INTRODUCCIÓN}

La insatisfacción por la política es, pues, en este sentido, no solo descontento por la propia política sino que sobre todo expresa la desproporción entre la autoridad oficial, que se presenta como política y es impotente, y una trasformación amplia de la sociedad que lenta, aunque constantemente, pierde capacidad de decisión y se encuentra marginada al terreno de lo no político. De ahí que los conceptos de lo político o no político queden imprecisos y requieran una revisión sistemática. ${ }^{4}$

A comienzos del siglo XXI, la actividad del Estado ha adquirido una revalorización tanto en las formas de organización de las instituciones gubermentales y sus estructuras y procesos, así como un fenómeno de estudio para las ciencias y humanidades. El Estado hoy no se entiende solo como un garante del orden y seguridad, propio del pensamiento liberal clásico, sino un actor con presencia de gran alcance en la intervención de la economía y formas de penetración vía políticas sociales, culturales, etc. en la sociedad, organizaciones y grupos sociales de los más diversos. Esto amerita por tanto procesos de investigación inter y transdiciplinarios para explorar, describir, analizar y evaluar los alcances de estos procesos políticos. Así es como nace este artículo, como una forma de hacer operativa las relaciones entre académicos de universidades del sur de Chile por investigar en cuestiones ligadas al profundizar la democracia subnacional, desarrollo político en el territorio, los procesos de descentralización y desconcentración, la estructura políticoadministrativa del país y los fenómenos políticos subnacionales en Chile.

El proceso político chileno de inicios de siglo ha estado marcado tanto por la herencia de la dictadura militar así como por las dinámicas y reformas acahecidas en los 90, derivado de la transición democrática en Chile, y que impulsó lo que se conoció como Crecimiento Económico con Equidad $^{5}$. A esto se suma la relevancia que adquiere en planos intelectuales como institucionales el territorio como variables de análisis y fenómeno de investigación en las ciencias sociales, lo que nos deja como principal legado para ese siglo dos grandes aportes, modelos teóricos que analizan las disparidades de desarrollo territorial que experimentan las naciones, así como la existencia de un proceso al interior de los científicos sociales por relevar la importancia por generar procesos de investigación aplicada a los fenómenos políticos y de política pública, utilizando para ello todo el desarrollo de la revolución estadística asistida por computadores, que permiten diseñar estudios

\footnotetext{
4 Ulrich Beck, "La Sociedad del Riesgo: hacia una nueva modernidad", en ediciones Paidós Ibérica, S.A., Barcelona, 1998, pág. 239.

${ }^{5}$ Más información en el ya clásico, Ffrench-Davis, R. (2003).
} 
para la toma de decisiones por mejorar la calidad de vida y el desarrollo de las sociedades en sus territorios ${ }^{6}$.

Algunas características contemporáneas frente al análisis de las cuestiones centrada en el desarrollo regional, son pesquizar los actores que deben intervenir, esto es, la sociedad civil, las autoridades públicas y los especialistas del mundo académico; ellos son los que tienen que tomar una decisión a la hora de analizar la política pública en marcha, bajo la premisa que los recursos financieros siempre son escasos a la hora de alcanzar mayores niveles de bienestar social y calidad de vida, así como insertarse en un proceso de desarrollo regional más equitativo e inclusivo (PNUD, 2015:116).

Es de interés de la presente publicación explorar el proceso político que ha dado origen a la nueva región de Los Ríos ${ }^{7}$, centrándonos en su origen, puesta en marcha y prospección. No nos da ahora hacer evaluaciones, menos considerando las renuencias de las autoridades políticas y administrativas del gobierno central y delegados de cada uno de ellos, que con el solo hecho de tematizar la evaluación se crispan; a esto se suma el escaso desarrollo científico - técnico en el campo de la evaluación de políticas y políticas públicas en contextos subnacionales en Chile.

Pretendemos un ejercicio académico entre la economía y la ciencia política por explorar modelos teóricos, metodológicos y de gestión sobre los procesos políticos subnacionales, teniendo como caso de estudio la región de Los Ríos, principalmente porque es un proceso de cambio político inducido por procesos y movimientos de la sociedad civil organizada, como el "Comité Nueva Región Provincia de Valdivia", y por tantas otras instituciones y personas anónimas que han sido partícipes de rediseños en el gobierno del territorio de la actual región de Los Ríos. Por lo mencionado anteriormente, esto lleva a plantear los objetivos de estudio que son, por un lado, analizar el proceso actualmente en marcha como un proceso de gobernanza y, por otro lado, determinar si del citado proceso se han creado las instancias políticas y administrativas que conlleve descentralización y la participación ciudadanía.

\footnotetext{
${ }^{6}$ Véase como ejemplo, Buzai, G. D., \& Baxendale, C. A. (2013).

${ }^{7}$ Para la creación de la Región, fue de vital importancia la ciudadanía valdiviana que se organizó rápidamente cuando en 1974 se instala el proceso de regionalización del país impulsado por el gobierno militar. Lo hizo a través de más de 60 organizaciones gremiales, sindicales, juntas de vecinos, deportivas, para hacer frente a lo que los dirigentes de la época vaticinaron sería un fracaso. Hoy seguimos considerando que esta División Política Administrativa (DPA), no ha cumplido con los fines para la que fue creada. Terminado el régimen militar, la ciudadanía se vuelve a reorganizar en lo que llamamos hasta hoy en día, Comité Provincia de Valdivia Nueva Región (1991), y debió bregar durante 17 años más para lograr la división territorial. Lo que parecía que con la democracia se lograría con mayor facilidad, no fue tal.
} 
Las preguntas del presente artículo son: ¿Cuáles son los antecedentes y procesos de puesta en marcha de la región y nuevo gobierno regional de Los Ríos? ¿Qué roles han jugado los actores políticos, sociales, económicos y académicos en la gobernanza del desarrollo del proyecto político subnacional de Los Ríos? y ¿Cómo se debiera avanzar en la consolidación del proyecto político territorial, económico, intercultural, social, científico y medioambiental?

Debemos recordar que la región de Los Ríos fue creada por Ley № $20.174^{8}$, dando origen tanto a la región como a la Provincia del Ranco. (Art. 1). Por ley, se crea la planta del Servicio Administrativo del Gobierno Regional con un total de 55 cargos y la planta del Servicio de Gobierno Interior con 18 cargos (art. 3-4). A esto se suman cargos en el Servicio Electoral, Ministerio Público, modificaciones en el Código Orgánico de Tribunales, Juzgados de Familia, Juzgados Laborales y de Cobranza Laboral y Previsional. (Art. 6-7-8-9-10). Además incluyo "procesos de reasignaciones presupuestarias en el 10 año" (Art. 12). La Ley entró en vigencia 180 días después de la publicación, fecha a contar de la cual se nombró al Intendentes y gobernador de Ranco (Art. 13). Además se identifica un proceso de traspaso de infraestructura, constitución del Consejo Regional de Los Ríos, recursos y gestión (Disposiciones transitorias). Finalmente, esto implica además ajustarse a la Ley № 19.175 Orgánica Constitucional de Gobiernos Regionales ${ }^{9}$.

En las disposiciones jurídicas con la creación de la región, se produjo un cambio en el mapa político-administrativo, nuestras fronteras regionales se modificaron y el nuevo territorio se conforma con dos provincias (Valdivia y del Ranco), tenemos el "contenedor". Se inicia la tarea de construir la nueva región y dar forma al "contenido", que desde el punto de vista ciudadano y el clamor del Comité Nueva Región Provincia de Valdivia, debe ser la región que soñáramos y no una réplica de las regiones ya existentes; de hecho, éste es el sentido más fundamental de crear una región nueva y no, una nueva región. Esto cobra relevancia para la ciencia política y la economía como modelo de proceso político regional que incide en las dinámicas del desarrollo económico y sociocultural.

Los autores asumimos los principios rawlsianos donde el Estado promueve justicia y equidad social, así como asumimos que los procesos de descentralización en Chile pueden ser entendidos en una doble hélice de cambios políticos y reforma administrativa-financiera de regiones, provincias y comunas. Particulamente las regiones son las estructuras idóneas para

\footnotetext{
${ }^{8}$ Biblioteca del Congreso Nacional (2007) Historia de la Ley № 20.174 Crea la XIV de Los Ríos y la provincia de Ranco en su territorio.

${ }^{9}$ Extracto de documento interno.
} 
distribuir la riqueza y buscar la eficiencia de los recursos y resultados en un entorno globalizado.

Compartimos los autores, lo que señalara Joan Prats, "la reforma institucional sólo es posible si además de buenas capacidades técnicas, que son imprescindibles, tenemos capacidad de conducción política de las reformas; es decir, si sabemos gestionar los conflictos, construir los consensos y expresa los nuevos modelos mentales que lleven a la convicción de que al final casi todos ganaremos más, la sociedad será mejor y hasta podemos conseguir, gracias al desarrollo, formas para compensar a los que pierden" (Prats, 2005:4).

En este contexto, puede decirse del punto de vista de Ocampo (2001:9-10), se reconoce que la liberalización económica que fue presentada al mundo como la respuesta a las estrategias ineficientes asociadas a la protección comercial, a los altos niveles de intervención estatal y a la captación de rentas (rent seeking) por parte de los agentes económicos, que dichas estrategias generaban. Fue presentada también como la forma de aprovechar plenamente las oportunidades que ofrecía la globalización. Esta visión representó un cambio significativo frente a la idea que sirvió de base a las estrategias de desarrollo durante varias décadas, según la cual la “industrialización tardía” exigía una amplia intervención del Estado.

Las actuales estructuras institucionales y las elites dan forma a un modelo de desarrollo político territorial en Chile convergentes con los sentidos operativos del sistema político nacional, restrictivo o no con las dinámicas democráticas a nivel subnacional (Gibson, 2006:222). Los antecedentes conceptuales están limitados a los actuales estudios en la ciencia política europea y americana sobre la política subnacional, como fenómeno de estudios interdisciplinarios sobre el territorio, la geografía y la planificación territorial.

Un fenómeno relevante para tratar de comprender el caso de Los Ríos, es lo referente a procesos de gobernanza sistémica, dado que se pone el acento en las capacidades de las instituciones de la sociedad civil y el empresariado por aportar la agenda, diseño, implementación, evaluación y promoción de las políticas que lleva a cabo los gobiernos. Mismo valor cobra en el enfoque de la competitividad sistémica. Acá en valor se encuentra en plantear como los encadenamientos productivos se dan en los territorios, por lo cual se hace necesario enfoques integrados de sistematización y análisis político

Dudas complementarias pretendemos abordar en posteriores publicaciones, derivado de la observancia del fenómeno de Los Ríos vinculado a su posicionamiento mundial. ¿Cómo la región de Los Ríos en un contexto de globalización? ¿Existirá convergencia o colisión entre nuestro patrón de desarrollo territorial y el mundial? Los autores asumimos que "es hora de comenzar a mirar hacia 
nuestras propias fronteras, más que estar todo el tiempo mirando hacia fuera" (Smith, Ph., y MaxNeef, M. 2011, 96).

Las interpretaciones que existen sobre los diferentes factores que se encuentran en el origen de las reformas descentralistas, han proporcionado interesantes elementos de análisis para dar cuenta de los propósitos y orientaciones políticas de estos procesos. Sin embargo, parece difícil que los procesos de descentralización puedan ser suficiente respuesta para dar solución a todos y cada uno de los problemas que se pueden revelar sobre el Estado, y la relación de éste con la sociedad civil ${ }^{10}$. "Asociatividad es el nombre del juego, pero para jugar se exigirá ahora a los jugadores conocimiento y autonomía; no es un juego para aficionados ni para subordinados"11.

La descentralización del Estado es una de las reformas políticas más recurrentes que los Gobiernos se han propuesto implementar en varios países latinoamericanos en las dos últimas décadas. Una breve revisión de lo ocurrido en la región respecto a ésta problemática en dicho período y, de acuerdo con Rosenfeld (1994:35), nos muestra que la mayoría de los países han iniciado procesos de descentralización, han promulgando nuevas leyes, o producidos cambios importantes a la legislación previamente existente sobre Gobiernos Regionales y Locales.

La hipótesis del presente artículo señala que:

"Aún cuando el Estado chileno es hiperpresidencial y centralizado, el caso de Los Ríos es una patrón singular de desarrollo político subnacional, que derivado de los usos por parte de los actores regionales de la legislación vigente, el proceso político administrativo, las dinámicas entre el ejecutivo, el Congreso Nacional y los entes subnacionales. Este caso es un tipo de reivindicación posdictadura de mayor autonomía regional, derivado de las reformas de los gobiernos regionales e instancias administrativas, por mejores mecanismos de gestión pública subnacional. Sin embargo, luego de esta fase, se hace necesario un nuevo modelo de desarrollo del territorio, que permita mayores niveles de gobernanza sobre la base de la proximidad a los problemas públicos, tanto dentro del contexto del Estado multinivel, así como en la implementación y evaluación co-responsable de las políticas y los procesos políticos".

\section{ANTECEDENTES DEL CASO}

Quizás la primera cuestión que debemos plantearnos sea una serie de hitos históricos, socioculturales, administrativos y políticos que dan cuenta de una historia de encuentros y

\footnotetext{
10 Véase por ejemplo en Rosenfeld, A. (1994). Para una exposición más extensa de enfrentar la descentralización a los gobiernos locales en América Latina.

${ }^{11}$ Boisier, S. (2004). Desarrollo territorial y descentralización. El desarrollo en el lugar y en las manos de la gente Revista Eure (Vol. XXX, № 90), pp. 27-40, Santiago de Chile, septiembre 2004.
} 
desencuentros, como se recoge MOP (2010:12), "en el que se nos han legado elementos de las poblaciones originarias mapuche, la presencia temprana y constante del elemento hispano, la incorporación de los colonos alemanes y otros desde la época republicana, como la mixtura y síntesis de cada una de estas influencias, sirve de base en que se desarrolló un territorio multicultural de la nueva Región de los Ríos, en la zona Sur de Chile". Desde una perspectiva histórica asociado a los pueblos indígenas, se destacan los trabajos de Guarda (1976:61) y Poblete (2008:55) que Valdivia se considera territorio mapuche-huilliche o huilliche aquel situado al sur del río Toltén, en este habita la sección meridional del pueblo mapuche, los mapuche-huilliches o huilliches. Esta división ha variado históricamente, pero se seguirá aquí, porque es la que suscita mayor consenso entre historiadores y antropólogos que estudian esta área.

De este modo, Poblete (2008:55) describe a la región de Valdivia (aproximadamente entre 1770 a 1848) que estuvo sujeta a cambios; los límites espaciales del antiguo Gobierno y luego Provincia de Valdivia estaban, al norte en el río Toltén, fijándose a principios del siglo XIX en el río Cautín o Imperial; el límite sur fue por mucho tiempo el río Bueno y a partir de 1796 el río Maipué (rio ubicado en la comuna de Purranque en su camino por la cuenca del río Negro y esteros que nacen en la vertiente oriental de la cordillera de la costa); el límite oeste el mar, y el este, la cordillera nevada. En 1797 Osorno pasó a depender del virreinato, hasta que en 1802 se reintegró, volviendo el límite sur al río Maipué. En 1826 se decretó una nueva división administrativa en la que se ratificó la antigua jurisdicción de Valdivia entre el río Toltén y el Maipué pero bajo el nombre de provincia. Finalmente, en 1861, cuando se formó la provincia de Llanquihue, el departamento de Osorno dejó de pertenecer a la provincia de Valdivia.

Relevante desde un punto de vista político histórico es el hecho que el territorio que comprende la actual región de Los Ríos ya a mediados del siglo XIX era considerado una unidad político - administrativa particular, lo que se reforzó con la oleada de nuevos habitantes y las previamente existentes primeras naciones. Generando un crisol de culturas, digna de futuros trabajos sobre capital social como un factor de desencadenamientos de procesos políticos como el que hoy exploramos.

En el año 1820, Lord Thomas Cochrane, capitaneando una pequeña escuadra integrada por la fragata O’Higgins, el bergantín Intrépido y la goleta Moctezuma, capturó las fortalezas de la bahía de Corral, para proceder luego a la toma de la ciudad de Valdivia, sellando con ello la vinculación de este territorio -y actual Región de Los Ríos- a la naciente República de Chile. En 1846 llegó a bordo del bergantín Catalina, el primer grupo de inmigrantes alemanes. Luego, en 1850, 
arribó a Corral el velero Hermann con 95 nuevos colonizadores germanos, entre ellos su líder Carlos Anwandter, quienes constituyen el símbolo cultural de la colonización alemana del sur de Chile.

Cabe, recordar, por otra parte, que el actual Paso Internacional Hua Hum ubicado en la Cuenca Lácar - Valdivia, Cordillera de los Andes, fue recomendado para su habilitación a Don Ambrosio O'Higgins, por el Coronel Ingeniero Juan Garland, remodelador de las fortificaciones Valdivia - Corral, para unir Valdivia con el Océano Atlántico. En torno a esta propuesta, el sabio Guillermo Frick en 1862, ubica el paso y gestiona su habilitación, iniciándose a fines del siglo XIX la penetración económica del puerto de Valdivia hacia la región Patagónica Argentina. Pero el proyecto no finaliza por dificultades financieras derivadas de la Primera Guerra Mundial.

Es importante destacar que, al igual que en el plano histórico-cultural, esta "el proceso de regionalización tal y como lo conocemos actualmente" tiene una larga historia, que está vinculada a variadas reformas a la división político-administrativa y de aspiraciones expresadas en terminar con el centralismo en sus diferentes dimensiones. Sus manifestaciones más recientes comienzan en el año 1967, bajo la presidencia de Don Eduardo Frei Montalva (1964-1970), se inició un proceso de regionalización en Chile como una manera de contribuir a potenciar los diferentes territorios del país, a través de la creación de la Oficina Nacional de Planificación (ODEPLAN). En esta ocasión, Valdivia constituía una región que abarcaba desde el norte la provincia de Cautín y por el sur incluía a la provincia de Osorno.

El año 1973, con la llegada al poder de la dictadura militar de Augusto Pinochet Ugarte (1973-1990), Chile experimenta un cambio en diferentes ámbitos, tanto políticos, económicos, sociales y culturales. En lo referente a los temas relacionados con el territorio, el nuevo Gobierno se enmarca dentro de una visión militar de defensa basada en el concepto de la geopolítica y, al mismo tiempo, en lo que se denominó la "doctrina de la seguridad nacional", que agregaba los sustentos políticos a un control de los territorios, sus ciudadanos y la defensa del país.

Como señalan los Documentos sobre Regionalización (1975:11), este proceso asumía la condición de Chile como un Estado unitario, "sin perjuicio de la plena vigencia de ello, la modernización del Estado de Chile por sus peculiares condiciones geográficas, requiere un sistema que permita un desarrollo descentralizado administrativa y regionalmente para que se materialice en la forma más perfecta posible la coordinación y la participación de las regiones en la función de Integración, la Seguridad, el Desarrollo socio-económico y la Administración Nacional” (1975:11). 
En 1974 pierde Valdivia su condición de capitalidad (hasta 1973 existían 25 provincias, entre ellas Valdivia, conformada por tres departamentos, Valdivia, La Unión y Río Bueno, cuya capital era la actual ciudad de Valdivia) y, se crea una nueva región conformada por las provincias de Valdivia, Osorno, Llanquihue, Chiloé y Palena; asignándose el rol de capital regional a la ciudad de Puerto Montt. Un territorio demasiado extenso para poder ser gobernado y que distanció definitivamente y de manera sustantiva a los ciudadanos de su gobierno regional. Valdivia pierde además en inversión pública, lo que impactó en el progreso de su población que desconoció las características históricas, políticas, geográficas y económicas de esta región.

Desde este momento y según Ramírez (2006, 7-8), se inicia un movimiento social, gremial y político en Valdivia con la creación del Comité Nueva Región Provincia de Valdivia que tensiona a la sociedad regional y se crean instancias de participación de amplia convocatoria transversal, en sus orígenes social y político que expresa la voluntad ciudadana de reclamar y recuperar el rol preponderante que la ex Provincia de Valdivia había tenido históricamente. Es así, como se crea un movimiento de sólidas bases sociales e institucionales constituido por la Asociación de Medianos y Pequeños Industriales y Artesanos de Valdivia, la Cámara de Comercio e Industria, Ilegando a agrupar y liderar a 42 organizaciones.

Para cambiar este panorama, ya de por sí desalentador, hay que notar que durante el gobierno del Presidente Patricio Aylwin Azócar (1990-1994) se realizaron un conjunto de reformas que establecieron los primeros mecanismos de gobernanza territorial, instancias de participación ciudadana que avanzó en el proceso de descentralización. Específicamente, con la publicación de la Ley Orgánica Constitucional sobre Gobierno y Administración Regional, entregando mayores atribuciones de autonomía a los gobiernos regionales. Este nuevo marco jurídico no solo posibilitó la primera elección indirecta de Consejeros Regionales, sino también, poder decidir por las iniciativas de inversión de desarrollo regional.

"Una vez que el panorama de reforma municipal estuvo resuelto, la atención
se centró sobre la propuesta de reforma regional. El componente político
más importante de esta reforma sería el establecimiento de los consejeros
regionales elegidos indirectamente. Al respecto, la Derecha política
manifestó su rechazo a la "politización" de los consejos regionales, que
resultaría si su generación estuviese encargada a los concejales municipales,
en vez de continuar en manos de los empresarios, profesionales y
trabajadores a través de los consejos de desarrollo regional (CODEREs)"
(Mardones, 2006:8). La modificación de estas tendencias exigían no más de los mismo. La profundización de las reformas eran sobre la base de nuevas visiones del proceso de regionalización, con renovados 
enfoques y distintos énfasis que bajo la presidencia de Eduardo Frei Ruiz-Tagle (1994-2000) se iniciaron importantes avances mediante la elaboración de diversos estudios científico-técnico sobre la viabilidad de la creación de la región, entre lo que se destaca la evaluación de la factibilidad de que la provincia de Valdivia se pudiera transformar en región en el año 1997 y denominado "Conflictos Regionales y Políticas Públicas". Además, en este contexto, uno de los hitos más relevantes fue el "Instructivo Presidencial al Gobernador de la Provincia de Valdivia". "Es importante tener en consideración que en los tres casos evaluados, se confronta a la posible nueva región con el conjunto de regiones existentes, esto para garantizar que ella cumpla el piso representado por el promedio del actual sistema de regiones, incluida la región de origen. En esta primera aproximación sólo Valdivia está por sobre el promedio de su conjunto, por lo que sería el único de los tres casos en que la petición entraría en la categoría de pre-aprobada" (Diagnóstico, 2003:22).

Sin embargo, y más allá del debate administrativo durante la presidencia de Ricardo Lagos Escobar (2000-2006), la creación de la Región de Los Ríos implicó una extensa tarea de acción colectiva que debió sortear las más diversas dificultades, entre ellas, la reforma a la Constitución Política de la República de Chile, debido a la existencia del numeral "13" en el Artículo № 45, que hacía referencia a la cantidad de regiones en el país, y a la ausencia de la palabra "regiones" en el Artículo № 99, que permitía sólo la creación de nuevas comunas y provincias en el territorio nacional. La dinámica del proceso de el cambiar los artículos 45 y 99 de la Constitución Política del Estado de Chile es aquellas que exige 4/7 de aprobación de los miembros en ejercicio de cada Cámara (Cámara de Diputados y Senado) aprobarla, modificarla o derogarla. Al igual que en el caso del estudio año 1997, le siguió otro estudio a solicitud de la Subsecretaria de Desarrollo Regional y Administrativo (SUBDERE) el año 2003, el que fue realizado por el Instituto de Geografía de la Pontificia Universidad Católica de Chile denominado "Diagnóstico y Propuestas para Modificar la División Político Administrativa del país". Ambos estudios coinciden en que la Provincia de Valdivia contaba con una serie de condiciones que hacían viable su construcción como región. Esto implica asumir un punto de vista eje del artículo, que la creación de la Región de Los Ríos fue un ejercicio político subnacional, de ahí la pertinencia como caso de estudio.

Entre estas experiencias y teniendo en cuenta todo lo anterior, tras un largo debate parlamentario permitió que el 26 de agosto de 2005, se promulgó la reforma constitucional contenida en la Ley № 20.050, que introdujo modificaciones a la Constitución Política de la República, entre otros, el actual artículo 49, a través del cual se eliminó el numeral referido a la 
cantidad de regiones y se uniformó el procedimiento para crearlas y suprimirlas, dejando entregado a una ley, de rango orgánico constitucional, lo relativo a la división políticoadministrativa del país. Esta importante modificación permitió que en octubre de 2005, en la ciudad de Valdivia, el Presidente Ricardo Lagos Escobar firmara el citado proyecto. Como describiera El Diario Austral de Valdivia el 19 de octubre del año, "En una fiesta ciudadana se transformó la firma por parte del Presidente Ricardo Lagos, del proyecto de ley que será enviado al Congreso para crear la Región de los Ríos, con lo cual comienza a ponerse fin a los 31 años de postergación administrativa de Valdivia", (Austral de Valdivia, 19/10/2005).

Ahora bien, un intento por clarificar los límites territoriales de la nueva región fue en el año 2006, cuando se comenzó a realizar el trámite constitucional sobre la división de la región de los Lagos, con el objetivo de la creación de la Región de Los Ríos, el gobierno central sugirió la incorporación de la provincia de Osorno a la nueva región. Sin embargo, esto fue rechazado por las autoridades locales, que propusieron la realización de una consulta ciudadana, con el fin de recoger la opinión sobre en qué región se encontraría el mejor futuro de la provincia. Fue así como el 19 de noviembre de ese año se realizó en Osorno una consulta ciudadana, la cual con más de 20.000 votos, solo un $6 \%$ se manifestó a favor, y un 92\% de los osorninos rechazó su incorporación a la nueva región. A pesar de que este plebiscito tenía en un comienzo carácter de no vinculante, el gobierno acogió y reconoció la importancia de dicha votación, por lo que decidió retirar del proyecto de ley la incorporación de la provincia a la futura Región de Los Ríos, permaneciendo la provincia de Osorno en la Región de los Lagos.

Por último, siendo relevante confirmar que la constitución de la XIV Región, Región de Los Ríos es el resultado de un largo proceso ciudadano por recuperar el estatus político-administrativo que tenía hasta 1974 la ex Provincia de Valdivia y corresponde a una nueva región de Chile, originada de la división de la actual X Región de Los Lagos en dos. Comprende la Provincia de Valdivia y la Provincia del Ranco. Esta última incluye a las comunas de La Unión, Futrono, Río Bueno y Lago Ranco. En tanto, la nueva Provincia de Valdivia quedó reducida y conformada por la comuna del mismo nombre, además de Mariquina, Lanco, Los Lagos, http://es.wikipedia.org/wiki/CorralCorral, Máfil, Panguipulli y Paillaco. En esta dirección, la creación de la Región de Los Ríos se formalizó con la promulgación de la Ley № 20.174 por parte de la Presidenta de la República, Michelle Bachelet Jeria (2006-2010), en Valdivia el 16 de marzo de 2007. Este hecho, además, se ha trasformado en uno de los hitos más relevantes en materia de 
descentralización intrarregional desde la instalación de los Gobiernos regionales, a inicio de la década de los 90, es representada a través de la siguiente figura.

Figura 1: Mapa de la distribución político-administrativo de la actual Región de Los Ríos.

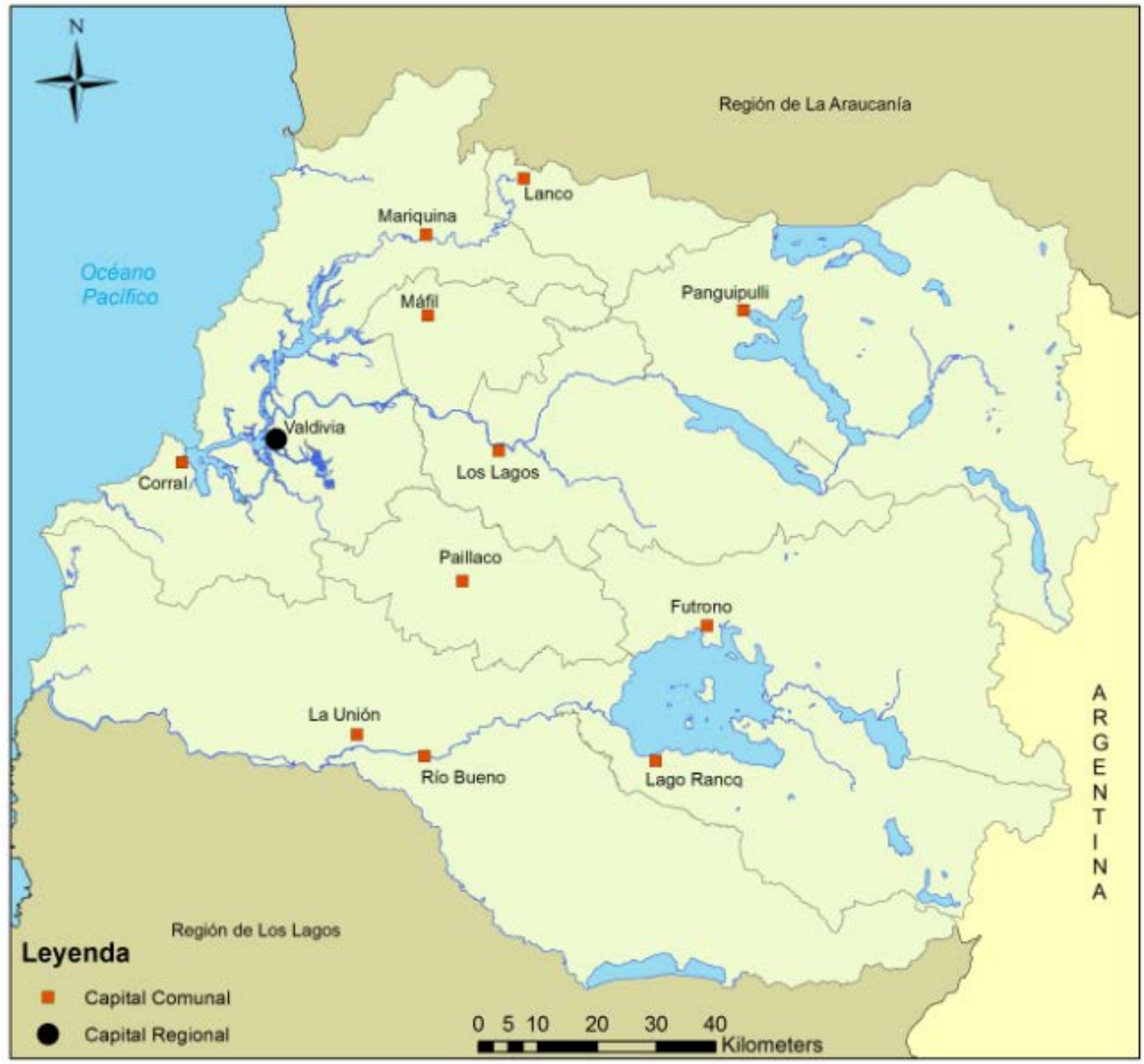

Fuente: Diseñado por Geógrafo Felipe Gutiérrez.

La Región posee una población de 329.925 Habitantes, de los cuales el 18 \% se identifica con el pueblo huilliche (los huilliche, son parte del pueblo mapuche que habita desde el río Tolten (Xolxenlewfu) al sur de Chile hasta, en la actualidad, el Cabo de Hornos, y tiene una dinámica distinta de organización tradicional, y se ubican principalmente en las comunas de Panguipulli, Lanco, Mariquina, la Unión, Futrono, Lago Ranko, Río Bueno, y en menor proporción en Valdivia, Corral, Mafil, Paillaco y Los Lagos.

En el próximo apartado de la presente publicación se pretende exponer una serie de argumentos sobre el proceso político llevado a cabo en la región de Los Ríos, a fin de articular 
enunciados que permitan explorar líneas para procesos posteriores de evaluación del proceso político, administrativo y organizacional de poner en marcha, desarrollar y consolidar un gobierno subnacional en nuestro país.

\section{PROCESO POLÍTICO DEL PROYECTO DE REGIÓN.}

La pertinencia del presente artículo surge con la finalidad de analizar la actual Región de Los Ríos conformada por las provincias de Valdivia y Ranco, teniendo como capital regional a la ciudad de Valdivia a ocho años de su creación, y por otro lado, ante este nuevo escenario en el proceso de regionalización del país para someter un análisis y seguimiento desde las demandas ciudadanas, como instrumento de control de gestión que haga posible una exitosa implementación y que asegure un desarrollo sostenible y equitativo, en todo el territorio regional. Es decir, si desarrollo se trata, nuestro proyecto de creación de "región nueva" debe ser un proyecto social inclusivo, equitativo y moderno, hacer gobierno, como uno de los pocos casos de desarrollo regional exitoso en América Latina (Boisier, 1998:8, 2003:33).

Para Moncayo (2002:9), la insurgencia de lo territorial se interpreta como una consecuencia de la crisis o vaciamiento del Estado-nación, piedra angular de la organización política del mundo desde la suscripción del Tratado de Westphalia en 1648. Por tanto, los cambios producen presiones para una redistribución de la autoridad tanto hacia arriba como hacia abajo, creando estructura que podrían ser capaces de responder de modo más efectivo. Esto implica que el Estado multinivel (Escolar, 2011:29), entendido como una red de articulación políticoadministrativa de organización del poder, ha sido una respuesta a los vaivenes del Estado latinoamericnao a fines del siglo XX e inicios del siglo XXI.

Por tanto, el desarrollo de la nueva región dependerá, al menos en parte, de las decisiones que tomen las autoridades competentes. Estas decisiones serán tanto más adecuadas y oportunas en la medida que se disponga de un mayor conocimiento de la realidad, es decir, de mejor información y de mayor número de técnicas que permitan su tratamiento y utilización. El principio de proximidad. Estas involucran nuevos desafíos para sus actores políticos, económicos y sociales, ya que son ellos los que poseen las habilidades y conocimientos necesarios, quienes formarán el capital humano que se requiere para impulsar el desarrollo de la región nueva, constituyan las condiciones para una gobernanza sistémica en la región.

Como se señaló al inicio del presente artículo, los rasgos del proceso de la Región de los Ríos, puede ser observado desde los modelos de la gobernanza territorial. Esta línea de 
investigación en las ciencias sociales, especialmente en el caso de la Ciencia Política, reviste de profunda relevancia para lograr entender los fenómenos emergentes de política subnacional. Si bien existe primacía de la política, hoy en día el poder político entra en diálogo con un sistema variado de actores que poseen otros tipos de atributos de poder. Será en esa coordinación de actores donde se asuma como acciones relevantes para la democratización subnacional, las mesas de trabajo desarrolladas, así como el diálogo auspiciado por los centros universitarios.

Sin embargo, cuando nos situamos en la arenas de las políticas, como señala Abarca (2002:100), supone hacer frente a tres posibles perspectivas generales de análisis; "análisis para las políticas públicas", la cual contempla el uso de técnicas estadísticas-matemáticas, instrumentos de evaluación, entre otros; "análisis retrospectivo de la construcción de algunas políticas a efectos de analizar su instrumentación". Y finalmente, "análisis del proceso político", que remite a los intereses en juego en la construcción de la agenda, la formulación de la política, y la adopción de las decisiones respectivas. De ahí que se haga necesario generar instancias universitarias de investigaciones evaluativa de la política y las políticas, así como la participación ciudadana. "Un elemento clave de este informe es que ciertos aspectos importantes de las políticas públicas dependen de manera fundamental de la habilidad que tienen los actores políticos para llegar a acuerdos intertemporales y para asegurar su cumplimiento, esto es, de su habilidad para cooperar. En entornos políticos que facilitan tales acuerdos, las políticas públicas tienden a ser de mejor calidad, menos sensibles a los shocks políticos y más adaptables a las condiciones económicas y sociales cambiantes" (Stein et al, 2006:20).

Sin duda, dos de los grandes desafíos y esperanzas para el proceso de regionalización chilena son, por un lado, alcanzar mayores niveles de decisión y participación ciudadana, por otro, el mejoramiento de la calidad de vida para todos los habitantes del territorio, más aún cuando éstos pasan de ser "habitante" a "ciudadano" del territorio. Este tema ha sido examinado ampliamente en el último tiempo en muchos debates y discusiones desde las ciencias sociales, como se recoge en Beck (1998; 238), cuando se da vueltas al calificar a la participación, a veces en tono amenazador y a veces esperanzador: para unos es una amenaza, para otros una esperanza. De acuerdo con este autor, éste es el compromiso hoy en día de la política pública y, por otro lado, la calidad y la determinación de criterios para evaluarla se han convertido en el gran tema. Desde los años noventa, en Chile, esta es una práctica de larga data. En esta línea argumental se sitúa la idea expresada por Meny y Thoenig (1998:98), sobre los objetivos perseguidos por la política, los sujetos afectados y los ejecutores (1998:98). 
Los proyectos políticos territoriales deben considerar la alta participación social en su elaboración, con un elevado nivel de representatividad, buscando y detectando a los líderes regionales de los más variados ámbitos, permitiendo una rápida o amplia socialización del tema que permita salir del elitismo que muchas veces congela en ciertos sectores de la sociedad las demandas y proyectos de carácter regional, restándoles fuerza y capacidad movilizadora. Un ejemplo de base social extendida, variada y con un ethos movilizador es Comité Ñuble Región, cuya fortaleza está en ese sentimiento de pertenencia, de clara identidad, con una cultura local altamente valorizada y en una conducción que parte de la sociedad civil, que veremos más adelante

Ahora bien, la lucha por los derechos a ser región tuvo consecuencias políticas serias para quienes lideraran este proceso. Como debe recordarse, Chile vivía bajo un gobierno autoritario, no obstante las amenazas que pesaban por parte del Gobierno sobre ellos, fueron capaces de continuar su trabajo. Se agrega a las organizaciones antes señaladas el Consejo de Desarrollo de la Provincia de Valdivia, constituido por destacados empresarios y organizaciones sociales. Por tanto, como resultado de estas luchas y demandas el Gobierno dicta un instructivo presidencial que otorga atribuciones especiales a Valdivia para influir en la toma de decisiones concernientes al desarrollo regional. Este instructivo no pasó de ser una mera formalidad que nunca tuvo repercusiones prácticas.

Cabe destacar que las características y la factibilidad de la nueva región, fueron analizadas también en un grupo de estudio de la Universidad Austral de Chile (UACh) que publicó tres libros ${ }^{12}$, concernientes a la conformación de la nueva región, así como la realización de seminarios y otras actividades que permitieron un amplio diálogo sobre este importante tema. Esperamos que esta opción por lo público que hemos tenido como Universidad Austral se profundice ahora que se hace necesario tantos procesos de evaluación de lo realizado, así como la generación de cuadros profesionales capaces de aportar el desarrollo del gobierno regional y de la región. Especial punto revista hacernos parte de los procesos de implementación de políticas, ya que se ha descrito en una serie de estudios sobre el tema en Chile y en América Latina, que el principal problema de las

12 En el año 2006: La nueva Región de los Ríos. Una mirada desde la universidad, en el año 2004: Construcción de Regiones: Desafí pendiente. Ambas Ediciones Universidad Austral de Chile y, en el año 2008 se realiza un workshop internacional en conjunto con el Instituto Nacional de Estadística, Región de Los Lagos que obtiene como resultado la publicación; Indicadores para el desarrollo: Una oportunidad para mirar la Región de los Ríos. Edita INE, Puerto Montt.

Revista de Direito da Cidade, vol. 08, no 2. ISSN 2317-7721 pp.538 -567 
políticas públicas sean sus mecanismos de operacionalización, esto es, en los medios para alcanzar los fines.

En ese contexto las universidades regionales tienen la función de contribuir a la creación y promoción de bienes públicos universales, pero más allá del proceso social en el que se enmarca, se liga muy estrechamente al cultivo y difusión del conocimiento, la cultura, la producción artística, la ciencia, la innovación y la tecnología, y a tareas específicas fundamentales como la descentralización y el desarrollo regional equilibrado. La universidad ha sido asociada también como un instrumento de promoción social por excelencia, otorgando a los sectores menos favorecidos la oportunidad de superar su condición, y al país la oportunidad de valorar el capital humano que servirá de base al desarrollo del país en su conjunto. Por eso no es de extrañar como por su misión, se vincula a la satisfacción de un interés colectivo, consistente en la realización del rol social de la Educación Superior en el desarrollo del país y de las regiones (UACh, 2011:13).

Oportuno es señalar que una vez dado inicio el proceso de instalación el primer gobierno de la región una vez constituida, fue elaborar una Estrategia de Desarrollo Regional de la Región de los Ríos (2009-2019), cuyo estudio de prospectiva fue encargado a la Universidad Austral de Chile. En él se destacan seis ejes temáticos definidos como estratégicos: territorial, social, económico, medio ambiental, científico-tecnológico. En este sentido del diagnóstico de base que se relaciona a los problemas asociados a ciertos ejes estratégicos y, que en orden de importancia están la calidad que tiene en la ciudadanía el rol y responsabilidad del sector público en el desarrollo de la región, la tendencia a concentración de población y actividades en la capital regional la ciudad de Valdivia; otro aspecto de gran relevancia y preocupación lo constituye el desarrollo sustentable, es decir la compatibilidad entre el desarrollo del bosque nativo, las plantaciones forestales, la producción de energía eléctrica y el turismo. Preocupación fundamental es la producción de energía y su vinculación a diversos sectores productivos ${ }^{13}$ Con la instalación de la nueva región de los Ríos se aprecia una mayor cercanía del gobierno con la ciudadanía, la distribución del presupuesto regional se hace cada vez más equitativo en los territorios llegando a grados altamente eficientes en el desembolso de los gastos del Gobierno Regional.

En el marco de estas reflexiones, las autoridades deberían fijarse como una de sus metas sobre las características que debería tener una región Modelo, como sería la Región de Los Ríos. Los principales elementos de la nueva región deben considera los siguientes: i) una identidad,

${ }^{13}$ Gobierno de Chile, Región de Los Ríos, Gobierno Regional: Estrategia Regional de Desarrollo. Región de los Ríos. 2009-2019, p.48-49. 
cultura, historia y sentido de pertenencia; ii) un sistema de centros poblados con amplia cobertura territorial y poblacional; iii) base económica compleja y sustentable orientada a la exportación; iv) acceso a la ciencia, tecnología y capital humano de alta calidad; v) una malla empresarial innovadora y competitiva; vi) liderazgos asociados con un proyecto político común que identifique y genere acuerdos en torno a tareas y objetivos compartidos; vii) fomentar una gerencia pública moderna y descentralizada; y viii) una inserción internacional creciente.

Finalmente, la movilización ciudadana al interior de la región por tan distintos temas adquiere especial importancia, los derechos fundamentales para nuestros pueblos originarios a tener en cuenta, frentes a intervenciones indeseables "desde arriba", dice relación con un factor de inicio de la recuperación de la memoria y cultura ha sido la reorganización del Logko Gvbam (Consejo de Logko). Desde su reorganización en marzo del 2001 el Consejo de Logko se ha presentado como una adecuada estructura que permite, en el contexto actual del desarrollo político y económico del país, proponer alternativas de desarrollo ante la actual intervención social y económica del Estado. En este marco, siguiendo al "Acuerdo de Cozoy Lofmapu", Comuna de Lanko, el día sábado 9 de enero de 2010, este proceso ha significado hacer aportes humanos y económicos para establecer una red de comunicación y conexión entre las reducciones y sus autoridades, y entre estas y las autoridades del gobierno, y entregar conocimientos (asesorías) para diseñar alternativas que puedan ser implementadas con el fin de recuperar su identidad cultural y espacios territoriales. En este proceso recuperar la memoria histórica es necesaria para la cohesión del pueblo, y por ello el Consejo ha visto la necesidad de realizar este trabajo de reconstrucción territorial para realizar propuestas de desarrollo coherentes con su visión de mundo.

Sin embargo como señala Le Bonniec (2013:29), "Ios Mapuche, en el caso de Valdivia e incluso de las provincias vecinas de Cautín, Malleco o Arauco, pocas veces han sido considerados como verdaderos sujetos políticos. Los escasos trabajos (Foerster \& Montecino 1988; Vergara, Foerster \& Gundermann 2005) que han dado cuenta de las relaciones que mantenían las organizaciones y dirigentes mapuche con las estructuras del Estado durante este período, se enfocaron más bien en la formación de una institucionalidad mapuche, mientras que el estudio de referencia de Correa, Molina y Yañez (2005) ha orientado su análisis hacia la confrontación entre estos distintos actores. Se mantiene, en consecuencia, un vacío historiográfico que particularmente destaca en el caso de la provincia de Valdivia, en cuanto a entender más profundamente las relaciones polimorfas que las distintas colectividades Mapuche entretuvieron con el Estado, y 
cómo, al mismo tiempo, participaron en la transformación de este y de sus relaciones".

\section{RESULTADOS Y DESAFÍOS.}

Luego de haber revisado tanto los antecedentes del caso y el proceso político observado en la Región de Los Ríos, es pertinente que avancemos en identificar líneas argumentativas sobre el futuro de este proceso político subnacional. Subyace para esto una mirada científica y académica sobre la posición y rol que debe tener el territorio de la Patagonia chilena, en una sociedad globalizada dinámica, plagada de circuitos de comunicación e interacción tanto económicos, como políticos, sociales y culturales. Las universidades son actores responsables institucionales y socialmente hablando, que colaboran en la construcción de regiones geográficas, políticas y productivas, que generan insumos y productos de investigación aplicada a las decisiones que toman las autoridades, políticos y funcionarios de la administración pública. Estas decisiones serán tanto más adecuadas y oportunas a medidas que se disponga de un mayor conocimiento de la realidad.

Consideramos que este caso en cuestión reviste características que hacen necesario una tercera fase de consolidación, luego de la creación y puesta en marcha. Este proceso por reconquistar una posición en el concierto nacional, como es ser región, plantea retos tanto para los propios actores involucrados, así como de manera indirecta evaluar los procesos de descentralización en Chile. Esto implica asumir con una universidad el compromiso por generar investigación básica como aplicada sobre política subnacional, posicionándose como un núcleo científico, que construye con otros actores del territorio, propuestas por vincular a la sociedad civil y actores gremiales con los procesos de modernización del Estado, más allá de lo logrado desde el reestablecimiento de la democracia en Chile.

Hemos planteado distinguir aspectos del proceso político, implementación de políticas públicas y aspectos jurídicos - administrativos, como forma de identificar cada uno de estos, instancias de análisis y proyección.

Podemos señalar hoy que tanto la ciudad de Valdivia como la Región de Los Ríos, han dejado de tener un rol secundario en el proceso de regionalización, derivado principalmente de la participación de los actores institucionales y de la sociedad civil. En la región se observa espacio de articulación entre los niveles de la institucionalidad a través de instrumentos actualmente disponibles para descentralizar a nivel local y buscar profundizar la participación ciudadana otorgando grados de control sobre las acciones de la autoridad regional y haciendo vinculantes las 
consultas comunitarias. Por su parte, las intervenciones para hacer frente a la falta de participación, durante el presente año podemos señalar el desarrollo de la "Mesa Portuaria Bahía de Corral 2014" y más reciente creación, en el día de las regiones, "Consejo de seguimiento de los compromisos de la Región Modelo". Es decir, como indica de la Maza (2008:15) a partir del texto del Comité de Modernización del año 2000, "hacer más eficiente los servicios públicos y fortalecer la democracia por medio de una gestión pública participativa" (De la Maza, 2008:15).

A esto se suma que el proyecto político subnacional posee aspectos identitarios marcados por la protección y sustentabilidad. La región de los Ríos tiene las condiciones por promover una propuesta de desarrollo sustentable que la convierta en región modelo, capaz de compatibilizar crecimiento económico, equidad social y protección de los recursos naturales. Este es un tema especialmente sensible para la población de la Región de los Ríos. Sin duda uno de los desafíos más importantes es la atracción de inversiones para la región que implique aumentar los empleos de calidad y de esta manera aportar a solucionar los problemas relacionados con la alta tasa de pobreza aun existente.

En cuanto a los procesos políticos no se puede estar atado a las dinámicas electorales cada cuatro años, se requiere de un sistema político con capacidad de responder a las demandas ciudadanas en tiempo real, robusteciendo las dinámicas institucionales regionales e intrarregionales. Es decir, pasar de un esquema de democracia liberal clásica a la chilena, hacia un sistema de representación más efectivo con mayor participación que representación. Esto implica profundizar prácticas de gobernanza territorial, participativa, que aumentadas con las nuevas tecnologías en el gobierno, permiten integrar en cada proceso de toma de decisión, análisis y evaluación, a los ciudadanos y ciudadanas de la región.

Como se expresa en la Estrategia Regional de Desarrollo de Los Ríos, eje estructurante de la efectiva política subnacional, sobre la finalidad del hacer gobierno "aquel que tiene como finalidad última el progreso permanente de la región y de la comunidad regional como un todo y de cada individuo, cuyo resultado es la interacción de un conjunto de procesos singulares, con énfasis en el crecimiento económico, la autonomía decisoria, la capacidad de captación y reinversión del excedente, inclusión social, conciencia ecológica, sincronía intersectorial y percepción de pertenencia"14(Estrategia Regional de Desarrollo Los Ríos, 2009:18) .

\footnotetext{
${ }^{14}$ Visión regional extraída de la Estrategia Regional de Desarrollo, aprobada por el Consejo Regional de Los Ríos en septiembre de 2010.
} 
Se puede decir que a futuro se ampliarán los espacios de participación ciudadana con la elección directa de los miembros tanto del Gobierno Regional (consejeros regionales) como del Intendente, ya que hasta ahora se realiza de manera indirecta y por designación directa del Gobierno central. Sin embargo, ante la nueva figura de "Gobernador Regional" propuesta en el proyecto de reforma constitucional, este actor ejercerá un control de las acciones del Intendente dentro de los temas del desarrollo regional, por tanto, hará más restrictivo la labor de la primera autoridad en el territorio, lo que además hace difícil la gestión del cargo. Aún así es prioritario definir una agenda política regional para los próximos años.

En el contexto de las políticas públicas que contribuyen a profundizar el proceso de desarrollo del gobierno regional y de la región, está en generar políticas que colaboren con la creación de valor en sectores tradicionales y emergentes, que permitan elevar la calidad de vida y mejorar la empleabilidad de los ciudadanos. Se requiere definir concentrarse estratégicamente en sectores en los que la región de los Ríos cuenta con un gran potencial, como: el turismo de intereses especiales; en el sector forestal, especialmente por la producción de celulosa, tableros y muebles; en el rubro alimenticio, por la producción de berries, lácteos, carne y verdura. Se debe recordar que la mayor producción de leche en el país se encuentra en esta región.

Por otro lado, es destacable el crecimiento significativo de las industrias creativas, sector económico-productivo rico en creación de valor, como el Festival Internacional de Cine de Valdivia que se ha convertido en uno de los más importantes de Chile.

"En los últimos años, la cultura ha pasado a formar parte de las estrategias de desarrollo local y regional y de las actuaciones de regeneración urbana, no solo por su potencial para generar riqueza y empleo, sino por su contribución a la diversificación del producto turístico, su capacidad para impulsar la cohesión social y el bienestar de los ciudadanos o su aporte a la diferenciación de los lugares. Los festivales culturales, una de las manifestaciones artísticas más dinámicas e interesantes del panorama cultural actual, también forman parte de las políticas de desarrollo económico, como consecuencia de las múltiples repercusiones económicas, sociales, culturales, turísticas o físicas - que generan en los territorios donde se celebran" (Devesa et al, 2012:111).

En el ámbito naviero, destaca la empresa ASENAV y su producción de embarcaciones a importantes mercados extranjeros como Noruega y Dinamarca. La empresa de fabricación de yates ALWOPLAST, con un alto estándar de tecnologías de punta, también se encuentra orientada a mercados externos. "Además han sido capaces de crear una imagen de su seriedad, calidad de sus construcciones y servicios, lo que les ha generado un importante prestigio a nivel internacional" (Oetinger, 2005:35). 
En el área de la educación, la Región de los Ríos posee un gran potencial por la presencia de universidades de prestigio, entre la que se encuentra la Universidad Austral de Chile, también existe un Centro de Estudios Científicos (CECS) dedicado a investigación de punta en el área de la biología, física teórica, glaciología, cambio climático y vinculado a los grandes centro de investigación del mundo. La educación y la innovación podrían constituir una especial apuesta al desarrollo de la región. Esto, dado a la cantidad de centros de enseñanza superior existentes que hacen de Valdivia una de las ciudades en Chile con mayor número de estudiantes de enseñanza superior por habitantes. En este ámbito, elevar la calidad de la educación es un asunto prioritario. Los niveles de la educación básica y media están por debajo de los promedios nacionales, según los instrumentos de medida que se aplican a los estudiantes a nivel nacional

En cuanto al ámbito de la conectividad, sigue pendiente y válida la bandera de lucha planteada por el Comité Nueva Región, en que señala un compromiso con Valdivia priorizando algunas iniciativas en pro de su desarrollo, entre las que se cuentan la licitación a privados del Puerto de Corral y el impulso al Paso Hua-Hum, como paso binacional y eje transversal estratégico que uniría el Océano Pacifico con el Océano Atlántico, entre la región y las provincias de Río Negro, Neuquén y Chubut de la República de Argentina. Impulsar el transporte extrarregional de productos a través del sistema portuario de la Bahía Corral, poniendo en valor su condición excepcional como: favorecer la navegabilidad del sistema fluvial del Río Valdivia, apoyar el desarrollo turístico de la bahía Corral vinculado a su sistema portuario y de navegación y, mejoramiento de infraestructura portuaria privada de Corral, entre otros. Se debiera diseñar un Corredor Binacional Valdivia - San Antornio Oeste - Viedma.

En cuanto a los aspectos asociados a normas, leyes, planes y estrategias de la administración pública el gobierno regional de Los Ríos a avanzado en ir generando marcos jurídicos ad hoc a las condiciones de la región, siendo uno de los principales avances la creación de la política regional de turismo, así como la constante observación de la Estrategia Regional de Desarrollo. En el contexto de acciones para-estatales la iniciativa "Nueva región cómo vamos" busca fortalecer las capacidades de la sociedad civil y autoridades locales, para avanzar en la construcción de una región más equitativa, abierta, democrática y sustentable, mediante la consolidación de un proceso emblemático en el país de construcción de una nueva región. Para ello, los objetivos específicos son los siguientes de la iniciativa (Miranda, J. y Bustamante, C. (2010:238): i. generar e instaurar un sistema ciudadano de seguimiento periódico y sistemático a los avances en la calidad de vida del territorio, mediante la evaluación rigurosa a la gestión pública 
y la promoción de una cultura ciudadana a la evaluación, ii. fortalecer el capital social mediante la generación de herramientas que fomenten y desarrollen capacidades y habilidades en la ciudadanía-organizaciones y funcionarios públicos- y que mejoren su participación e incidencia en la gestión de sus territorios y en los asuntos de interés público, y iii. promover el desarrollo sustentable de los territorios a partir del trabajo conjunto entre empresas y comunidad local, tendiente a mejorar la calidad de vida de estas poblaciones.

La particularidad del caso que hemos estado describiendo nos lleva a señalar, que a diferencia de lo describe Esteban Valenzuela (Valenzuela, 2016:243-244) en sus recientes Fondecyt, al estudiar casos emblemáticos de movimientos sociopolíticos en territorio, el único caso exitoso pero que excluye de su investigación, es la demanda regionalista que originó la Región de Los Ríos. En sus escritos, Valenzuela hace ver que los casos de la demandas de comunas del norte por el royalty sería uno, luego el movimiento mapuche en la Araucanía sería otro, y finalmente la demanda ecosocial de Aysén. A la luz de la evidencia es la región de Los Ríos el caso exitoso, sin embargo, esto no implica tener asegurado el éxito del proyecto político, pero si tener las capacidades institucionales, de los centros universitarios, gremios y sociedad civil organizada que presionen y se sumen en la implementación del proyecto político subnacional de Los Ríos. Será necesario en un futuro trabajo, comparar casos de política subnacional en Chile, así como en el conosur latinoamericano, como forma de generar una línea investigativa en los centros unversitarios.

Como forma de sintetizar un incipiente análisis político, se puede señalar a grandes razgos que estamos en presencia de un tema de gobernanza territorial, en la que han participado una serie de actores institucionales y no institucionales. Además estamos ante un hecho que ha logrado convivir con una Estado hiperpresidencial y centralista, comprendiendo las dinámicas de las políticas en Chile, cargadas con un fuerte acento en la gradualidad de los procesos.

Una tercera fase de consolidación requiere generar un pacto con el Estado central de tranferir competencias administrativas que permitan una mayor articulación entre el nivel regional y los municipios. Además requiere generar los instrumentos necesarios para poder pensar y desarrollar una región vista en el contexto mundial, siendo un polo atractivo por su propio modelo de desarrollo territorial. Esto implica diseñar proyectos para desarrollar los pasos de Carirriñe y Hua-hum, generando estrategias binacionales de atracción y endogenización de procesos economícos en el territorio.

El preciso generar una conciencia ciudadana basada en la democracia participativa y el 
fomento del desarrollo sustentable en todos los niveles del sistema. Solo de esa forma se obtendrá la anhelada armonía sociocultural y económica; y con ello un mejor sistema de ordenamiento territorial (Sanchez, 2008:104).

Desde nuestro punto de vista se hace imperioso desarrollar en nuestros espacios universitarios instancias de discusión, diseño y propuesta de estudios y tranferencias de capacidades, tanto en los procesos de investigación en el ámbito de los procesos políticos, la implementación de políticas públicas en condiciones subnacionales, el diseño de normas y reglas del juego para un desarrollo que integre a los actores del desarrollo. Así mismo, la Corporación Valdivia Ciudad Universitaria y del Conocimiento integrada por la Universidad Austral de Chile, Universidad Santo Tomas, Universidad San Sebastián y el Centro de Estudios del Sur, que deben promover al interior de sus espacios instancias de deliberación ciudadana sobre el proceso llevado a cabo, acompañando a los actores de la sociedad civil en la promoción y pertinencia de sus propuestas, así como instancias de evaluación periódica del estado de avance de las promesas de desarrollo regional.

\section{CONCLUSIONES.}

Repasar el proceso de reinstauración del gobierno subnacional de los territorios comprendidos en la actual Región de Los Ríos, nos da luces y sombras de itinerario llevado a cabo, pero con la seguridad que se trata de un proceso particular de desarrollo político subnacional.

Si bien quedan cabos sueltos en la puesta en marcha y desarrollo, no es menos relevante que este fenómeno es un ejemplo de la puesta en marcha de rediseños institucionales subnacionales, utilizando para ello las reglas del juego del modelo constitucional chileno, su lógica gradualista y la capacidad de implementar el gobierno central ventanas de oportunidades y concertación de actores para un buen desarrollo de la propuesta, puesta en marcha y desarrollo del gobierno regional, la administración del territorio y los efectos en el desarrollo regional.

Con esto podemos señalar que respondemos de manera satisfactoria a una de las preguntas de investigación, asociada a dar cuenta de los antecendetes del caso. El territorio de Valdivia, relativamente semejante a la actual región de Los Ríos, fue reconocido por la República como una instancia de adminsitración subnacional en el chile decimonónico, reafirmado ya en el siglo XX con un rediseño de las instancias de gobierno subnacional como eran las Provincias en Chile. Será solo el modelo implementado por la dictadura de Pinochet el que congele el desarrollo endógeno, supreditando este a lo definido desde Puerto Montt. 
Los Ríos en un caso divergente a lo expuesto por una serie de alegatos actuales por generar modelos de mayor autonomía y profundizar de manera general procesos de descentralización. Compartimos la necesidad de mayor descentralización, pero visto lo que ocurre en Los Ríos, hay dinámicas de asociatividad y concertación de actores que han permitido generar gobernanza. Esto implica asumir que el Estado chileno, más que generar grandes proyectos centralmente planificados, debiera entrar en interacciones con cada uno de los espacios subnacionales, regionales, provinciales y locales, caso a caso a fin de definir procesos por la tranferencias y competencias administrativas, una que otra política y ciertas decisiones financieras donde la autoridad recaiga en el sistema político-institucional regional.

Los aspectos relevantes en la construcción de la región en marcha, debemos situarnos en relación de los cuatro actores involucrados en el proceso de instalación de la nueva región y, el rol que han jugado, debemos decir: Qué el sector público destacó en el armado del aparato de Gobierno y Administración Regional con evidente y publicitado cuoteo político y el centralismo acostumbrado. Qué la ciudadanía con perseverancia y transversalmente logra la creación de la Nueva Región y apura una participación más efectiva y vinculante. No se le reconoce el rol en la consecución de la Nueva Región y no ha sido considerada en términos prácticos en la construcción de ella, y qué el sector privado ha tenido una mínima participación y solo maximizando sus esfuerzos cuando sus intereses económicos pueden verse afectados. El sector científico un actor activo en el diseño institucional del proyecto de nueva región, siendo los casos de casas universitarias tanto de la propia región, Universidad Austral de Chile, así como universidades de la capital. Falta sin embargo, asumir un rol fundamental de las universidades hoy en el contexto de la gobernanza, ser actores que promueven (advocacy) acciones que benefician a una sociedad en su conjunto, siendo por ejemplo un tipo de acción, ser parte de redes y secretarías técnicas de la gestión de las mismas redes.

Lo analizado en este trabajo, tiene que ver "cómo se hace un buen gobierno y alcanzar una democracia efectiva", no es solo resorte sobre si más o menos Estado sino más bien, cómo se interviene en las realidades para producir ciertos cambios en términos de los esperado por los ciudadanía. En este sentido a partir de las oportunidades, potencialidades y ventajas comparativas y competitivas sobre los procesos de regionalización y descentralización, es cómo estructurar acciones que conduzca a la solución tanto a las demandas sociales como a las técnicas que responden al análisis del entorno regional.

¿Cómo avanzar? Esta quizás deba ser una pregunta que solo sea posible de responder 
observando las dinámicas de los actores de la región, así como de las autoridades nacionales. Lo que si queda claro es que desde un punto de vista de nuestro enfoque se ha avanzado, pero se hace necesario profundizarlo que conlleve entrar en una face de consolidación del proyecto político subnacional.

Es imperioso contar con una memoria institucional, datos, documentos instituiconales, actos administrativos y proyectos que permita disponer de información en tiempo real información para una planeación anual multisectorial, mesas de trabajo con municipios, a fin de endogeneizar el proceso. El análisis de la factibilidad de las iniciativas impulsadas hasta ahora requiere tanto de una voluntad política clara y vinculo o requerir una modificación legislativa, así como la definición de agendas políticas regionales, en materias de sustentabilidad, ciudadania y transferencia de competencias. En esta dirección, desde los movimientos ciudadanos se aspira y propende apoyar políticos que cumplan con el requisito del voto programático y vinculado a la posibilidad revocatorio del mandato; restricción a ser reelegidos para evitar tanto candidaturas perpetúas como a clientelismo político; existencia de plebiscito regionales y locales; una nueva constitución; planificar el desarrollo regional según las características de la matriz productiva y; captar parte del impuesto que se genera en la región y no donde se declara.

La hipótesis del artículo se valida, sin embargo se requiere profundizar en casos comparados. La nueva gobernanza desde la gestión pública para alcanzar un mayor desarrollo, debiera fundamentarse tanto en la participación ciudadana como en todos los niveles de la sociedad civil, con la finalidad de conformar un orden social armónico en la región, bajo un modelo de cuatro actores: Gobierno, Sociedad Civil, empresariado y los centros universitarios, donde se distribuyan responsabilidades y deberes, como también existan obligaciones compartidas.

Sin embargo, queda pendiente como se articula este proceso con las reivindicaciones mapuches en la región, ya que el desarrollo requiere dinamizar el territorio. "Las lógicas de acción de los Mapuche en materia de reivindicación territorial, su anhelo por "recuperar las tierras" de sus antepasados, entraron en conflicto con otras aspiraciones de entregar "la tierra para quien la trabaja" (Le Bonniec 47:2013)

Somos concientes desde los estudios del desarrollo regional, que los procesos en el territorio ayudan a generar procesos de reequilibrios intra y extrarregional, implicando entre otras cosas la implementación de políticas de desarrollo político subnacional, que contribuyan a la profundización de la democracia. Esto es descentralización que genera participación, oportunidades y bienestar para Los Ríos, esto es, un proyecto político subnacional. 


\section{REFERENCIAS}

ABARCA, A. (2002). Las políticas públicas como perspectiva de análisis. Revista Ciencias Sociales 97:95-103.

ALBI, E., GONZÁLEZ-PÁRAMO, J., y LÓPEZ, G. (2000). Gestión pública: Fundamentos, técnicas y casos. Editorial Ariel Economía, Barcelona.

ALTIMIR, O. (2009). Distribución del ingreso e incidencia de la pobreza a lo largo del ajuste. Revista de la Cepal, 98, diciembre.

AMPUERO, R. (2004). La reforma regionales pendiente: Una condición sine qua non para la gobernabilidad democrática. Construcción de Regiones: Desafío pendiente. José Escaida y Juan Carlos Ferrada (editores). Universidad Austral de Chile, Valdivia, Chile.

AMTMANN, C. (2004). La Universidad Austral de Chile y su compromiso con la región. Construcción de Regiones: Desafío pendiente. José Escaida y Juan Carlos Ferrada (editores). Universidad Austral de Chile, Valdivia, Chile.

BECK, U. (1998). La sociedad del riesgo: hacia una nueva modernidad. Paidós Ibérica.

BIBLIOTECA DEL CONGRESO NACIONAL (2007) Historia de la Ley № 20.174 Crea la XIV de los Ríos y la Provincia de Ranco en su territorio. D. Oficial 5 de abril 2007. Valparaíso.

BOISIER, S. (1998). Teorías y metáforas sobre desarrollo territorial. Revista Austral de Ciencias Sociales, 2, 5-18.

(1999). Teorías y metáforas sobre el desarrollo territorial. Edita, CEPAL, LC/G.2030-P, mayo, Santiago de Chile.

(2003). El desarrollo en su lugar: (El territorio en la sociedad del conocimiento). Universidad Católica de Chile, Facultad de Historia, Geografía y Ciencia Política, Instituto de Geografía.

BUZAI, G., Y BAXENDALE, C. (2013). Aporte del análisis geográfico con Sistema de Información Geográfica como herramienta teórica, metodológica y tecnológica para la práctica del ordenamiento territorial. Persona y Sociedad. Universidad Alberto Hurtado, Vol. XXVII/N 2/ mayoagosto 2013/pp. 113-141.

CASTELLS, M. (2005). Globalización, desarrollo y democracia: Chile en el contexto mundial. Edita Fondo de Cultura Económica, Santiago, Chile.

COPER, S., MARTíNEZ, F. Y AZURMENDI, H. (2004). Construcción de Regiones: Desafío pendiente. José Escaida y Juan Carlos Ferrada (editores). Universidad Austral de Chile, Valdivia, Chile.

DE LA MAZA, G., (2008). Mecanismos de Participación Ciudadana en el Diseño, Implementación y Evaluación de Políticas Públicas (Ref: SCT/2008/5). Programa Ciudadanía y Gestión Pública Universidad de Los Lagos - Sede Santiago, Santiago de Chile - Julio. 
DEVESA, María, et al. Repercusiones económicas y sociales de los festivales culturales: el caso del Festival Internacional de Cine de Valdivia. EURE (Santiago), 2012, vol. 38, no 115, p. 95-115. DIARIO AUSTRAL de Valdivia (2005), Noticia sobre creación de la región. 19/10/2005.

ESCAIDA, J. (2004). Construcción de Región. Algunos antecedentes y desafíos pendientes. Construcción de Regiones: Desafío pendiente. José Escaida y Juan Carlos Ferrada (editores). Universidad Austral de Chile, Valdivia, Chile.

ESCOLAR, M. (2011). Nacionalización, comunidad cívica y coordinación electoral: Problemas para la integración del sistema político en estados democráticos multinivel. Revista Saap, 5(2), 0-0.

FERRADA, J.C. (2004). Descentralización administrativa, reformas constitucionales y Nueva Región de Valdivia. Construcción de Regiones: Desafío pendiente. José Escaida y Juan Carlos Ferrada (editores). Universidad Austral de Chile, Valdivia, Chile.

FFRENCH-DAVIS, R. (2003). Chile, entre el neoliberalismo y el crecimiento con equidad. Nueva Sociedad, 183, 70-90.

FUENTES, R Y MIES, V. (2005). Una mirada al desarrollo económico de Chile desde una perspectiva internacional. Revista Economía Chilena, Volumen 8-№1/ abril.

GIBSON, E. (2006). Autoritarismo subnacional: estrategias territoriales de control político en regímenes democráticos. Desafíos, 14, 203-237.

GOBIERNO DE LOS RÍOS (2010) Estrategia Regional de Desarrollo, aprobada por el Consejo Regional de Los Ríos en septiembre de 2010.

GUARDA, G. (1976). La Economía en Chile Austral antes de la Colonization Alemana. 1645-1850. Valdivia, Universidad Austral de Chile.

GUTIERREZ, Eric Iturriaga, and Francisco Vega Duarte. "Marco jurídico y prácticas públicas en Gobierno Regional de La Araucanía." Revista de Estudios Políticos y Estratégicos. 2.2 (2014): 10-33.

LE BONNIEC, Fabien. La participación de las comunidades mapuche-huilliche en el proceso de la Reforma Agraria en la Provincia de Valdivia (1970-1973). Rev. austral cienc. soc, 2013, no 24, p. $27-$ 49.

MARDONES, Rodrigo. Descentralización y transición en Chile. Revista de ciencia política (Santiago), 2006, vol. 26, no 1, p. 03-24.

MARINOVIC, E. (2004). Valdivia Región: Una lucha permanente. Construcción de Regiones: Desafío pendiente. José Escaida y Juan Carlos Ferrada (editores). Universidad Austral de Chile, Valdivia, Chile (pp. 67-86).

MAX-NEEF, M. (2006). Fifty key thinkers on development. Edited by David Simon, Routledge, New York. 
MAZA DE LA, G (2008) Mecanismos de participación ciudadana en el Diseño, Implementación y Evaluación de Políticas Públicas. Programa Ciudadanía y Gestión Pública.

MENY, I., y THOENIG, J.C. (1992). Las políticas públicas. Editorial Ariel S.A., Barcelona, España.

MINISTERIO DEL INTERIOR - Subsecretaría de Desarrollo Regional y Administrativo (2015) Decreto con Fuerza de Ley 1-1975. Texto refundido, coordinado, sistematizado y actualizado de la Ley № 19.175 Orgánica Constitucional sobre Gobiernos y Administración Regional. Santiago de Chile.

MIRANDA, J. (2004). Evaluación de las Políticas públicas: Accountability. En Escaida, J., y Ferrada, J.C. (comp.). Construcción de Regiones: desafíos pendientes (pp.37-47). Edita Universidad Austral de Chile.

MIRANDA, J. Y BUSTAMANTE, C. (2010). Participación ciudadana en época de crisis: caso de la Región de Los Ríos (Chile). En Edemir de Carvalho (organizador). Perspectivas da Globalização: E das suas contradições no Brasil e na América Latina (pp. 234-260). LCTE Editora.

MIRANDA, J.C. Y RAMOS, C (2007). La matriz insumo-producto para la región de los ríos: una tarea pendiente. Comunicación presentada en el Workshop Internacional Indicadores para el desarrollo: una oportunidad para mirar a la Región de los Ríos. Edita Universidad Austral de Chile, Valdivia (Chile), junio de 2007.

MONCAYO, E. (2002). Nuevos enfoques teóricos, evolución de las políticas regionales e impacto territorial de la globalización. Edita ILPES-CEPAL, LC/L.1819-P, Santiago de Chile.

MOP (2010). Diagnóstico Patrimonio Cultural Región de Los Ríos. Edita MOP de Los Ríos, Valdivia.

OCAMPO, J. A. (2011). Macroeconomía para el desarrollo: políticas anticíclicas y transformación productiva. Revista de la CEPAL, (104), 7-35.

OCAMPO, J.A., y MARTíN, J. (editores) (2003). Globalización y desarrollo: Una reflexión desde América latina y El Caribe. Edita Comisión Económica para América Latina (CEPAL), Bogotá, Colombia.

OETINGER L (2005) Diagnóstico y análisis de la Industria Naval en la Comuna de Valdivia. Tesina de grado. UACh.

OSORIO, S. (2010). "John Rawls: Una teoría de justicia social su pretensión de validez para una sociedad como la nuestra". Rev.relac.net.estrateg.segur.vol.5 n1, Bogotá Jan/June.

PNUD (2015). Desarrollo Humano en Chile 2015: Los tiempos de la Politización. Edita Programa de las Naciones Unidas para el Desarrollo, Santiago, Chile.

POBLETE, M. P. (2008). "Mapuche-huilliches e hispanocriollos en Valdivia. Cartas de petición y procesos de articulación en el período colonial tardío". Revista CUHSO, volumen 15 № 2.

PRATS, J. (2005) Desbloquear la política. Fundación Boliviana para la Democracia Multipartidista. 
(2009). Chile será descentralizado, o no será desarrollado. En Heinrich von Baer (Editor General): Pensando Chile desde sus Regiones (pp. 11-15). Ediciones Universidad de la Frontera, Temuco, Chile.

RAMIREZ, M. (2006). La Provincia de Valdivia. Una nueva región por la razón y la fuerza de movilización de sus habitantes, en La nueva Región de los Ríos. Una mirada desde la universidad. José Escaida y Juan Carlos Ferrada (editores). Universidad Austral de Chile, Valdivia, Chile (pp. 716).

ROSENFELD, A., (1994). Los gobiernos locales en América Latina. Publicado en la Biblioteca Virtual TOP.

SÁNCHEZ, Rafael, Las nuevas regiones de Arica y Parinacota y de Los Ríos, Chile. Algunos antecedentes sobre su estructura y funcionamiento (2009). Revista Geográfica Venezolana. Vol. 50 (1). 87-107.

SIERRA, E. (1994). La Pirámide en conflicto: pobreza, neoliberalismo y respuestas. Ediciones Cultura y Didáctica S.A. Quito, Ecuador.

SMITH, P. B., \& MAX-NEEF, M. A. (2011). Economics unmasked: from power and greed to compassion and the common good. UK: Green Books.

SMITH, PH., y MAX-NEEF, M. (2011). Economics Unmasked. Green Books, Cornwall, UK.

STEIN E, TOMMASI M, ECHEBARRÍA K, LORA E Y M. PAYNE (2006). La política de las políticas públicas. BID Ed. Planeta Mexicana, México.

SUBDERE - PUC (2003) Diagnóstico y propuesta metodológica para modificar la división político administrativa del país. Informe Final.

SUBDERE (1975), Documentos oficiales sobre regionalización, compilación realizada con ocasión de la reunión de Intendentes Regionales celebrada los días 17-18-19 de marzo de 1975. Santiago de Chile,

UACh (2011). Structurum Renovatio, Plan Estratégico 2012-2015.

VALENZUELA, Esteban; PENAGLIA, Francesco y BASAURE, Lía. Acciones colectivas territoriales en Chile, 2011-2013: de lo ambiental-reivindicativo al autonomismo regionalista. EURE (Santiago) [online]. 2016, vol.42, n.125, pp. 225-250. ISSN 0250-7161.

VEGA, F. (2013) en Monje-Reyes, P., \& Tenório, F. G. (Eds.). (2013). Ciudadanía, territorio y políticas públicas: pensando el desarrollo con democracia y ciudadanía territorial. Análisis de casos de Chile y Brasil.

VON BAER, H. (2009). Pensando Chile desde sus regiones. Temuco: Universidad de La Frontera. 
WILLIAMSON, J. (1997). "The Washington Consensus revisited", en Louis Emmerij (comp.), Economic and Social Development into the XXI Century, IDB, Washington, D.C., The Johns Hopkins University Press.

Trabalho enviado em 20 de janeiro de 2016.

Aceito em 20 de março de 2016. 